\title{
Weitere Hinweise auf Zusammenhang
}

Tierversuche zeigen, dass bei einer Insulintherapie die Serumspiegel des Hormons sowie des IGF-1 (insulin-like growth factor 1) deutlich steigen. Beide Faktoren sind in Tiermodellen an der

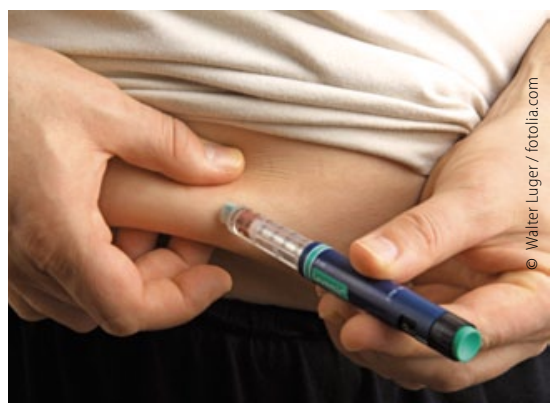

Eine Insulin-Dauertherapie scheint das Risiko an Krebs zu erkranken deutlich zu erhöhen.
Krebsentstehung beteiligt. Auch immer mehr Humanstudien weisen auf eine Assoziation zwischen einer Insulin-Therapie und der Entstehung einer Krebserkrankung hin. Eine aktuelle Metaanalyse von 15 Studien stützt die Vermutung. Insgesamt wurden Daten von fast 580.000 Studienteilnehmern ausgewertet.

Bei ausschließlicher Betrachtung der Ergebnisse von Fall-Kontroll-Studien hatten Diabetiker mit Insulintherapie eine $83 \%$ höhere Wahrscheinlichkeit für eine Krebserkrankung als Patienten mit einer antidiabetischen Therapie ohne Insulin. Werteten die Wissenschaftler nur Kohortenstudien aus, lag das Zusatzrisiko bei $28 \%$. Bei Berücksichtigung beider Studienarten betrug der Risikoanstieg $39 \%$.
Es zeigte sich kein Zusammenhang zwischen Insulintherapie und der Entstehung von Brust-, Prostata- und Leberkrebs, dagegen aber zwischen der Therapie und einem Pankreaskarzinom und einem kolorektalen Karzinom. Mit einem relativen Risiko (RR) von 4,78 - also einer fast 5-fach erhöhten Erkrankungswahrscheinlichkeit - war die Assoziation mit Tumoren der Bauchspeicheldrüse signifikant stärker als mit einem kolorektalen Karzinom (RR 1,5). Parameter, die solche statistischen Auswertungen am stärksten verzerren, sind Patientenalter, Geschlecht und der Bodymass-Index. Wurden jene Studien für eine Auswertung zusammengefasst, in denen diese Parameter herausgerechnet wurden, blieb die Assoziation zwischen Insulintherapie und Krebs bestehen (RR 1,81).

Peter Leiner

Janghorbani M et al. Systematic Review and Meta-analysis of Insulin Therapy and Risk of Cancer. Horm Cancer. 2012;3(4):137-46.

\section{Diabetikerinnen profiteren zweifach}

\section{Weniger Mammakarzinome unter Metformin-Therapie}

Diabetikerinnen, deren Blutzuckerspiegel mit Metformin eingestellt ist, erkranken seltener an invasivem Brustkrebs als Patientinnen, die andere Antidiabetika einnehmen. Das ergab eine Metaanalyse von sieben Beobachtungsstudien aus den Jahren 2009 bis 2012.

Nananda F. Col, Biddeford, ME/USA, und Kollegen wählten aus mehr als 400 Publikationen sieben Studien aus: vier Kohortenstudien und drei Fall-KontrollStudien. Das Risiko, an invasivem Brustkrebs zu erkranken, war näherungsweise um $17 \%$ niedriger, wenn die Patientinnen mit Metformin behandelt wurden (kombinierte Odds Ratio [OR] 0,83, 95\%-Konfidenzintervall [95\%-KI] 0,710,97).

Berücksichtigten die Forscher in ihrer Analyse nur die Studien, die mindestens über drei Jahre verliefen bzw. die, die vor vor 1995 begannen, war der Zusammenhang noch eindeutiger (OR 0,75; $95 \%$-KI 0,62-0,91 bzw. OR 0,68, 95\%-KI 0,55$0,84)$.

Dass gerade die langfristige Metforminbehandlung bei Diabetikerinnen mit einer niedrigeren Inzidenz an invasiven
Brustkrebserkrankungen assoziiert war, nehmen die US-Gesundheitsforscher als Bestätigung für den für den positiven Einfluss der Metformin-Behandlung, auch wenn sie einschränken, dass es sich bei den berücksichtigten Studien um Beobachtungsstudien handelte, die mit Verzerrungen einhergehen können.

Dass die Metforminbehandlung einen Einfluss auf Brustkrebs hat, wurde erst kürzlich wieder gezeigt: So war der Biomarker für die Tumorproliferation Ki-67 bei Mammakarzinom-Patientinnen, die über zwei Wochen Metforminim eingenommen hatten, deutlich niedriger als bei Frauen ohne Metformin-Therapie [Dowling RJ et al. J Mol Endocrinol.2012; 48:R31-R43].

In einer anderen Studie zeigten Diabetikerinnen, die an Brustkrebs erkrankt und auf Metformin eingestellt waren, war der Anteil der Patienten mit komplettem Ansprechen auf die Chemotherapie signifikant höher als bei den Diabetikerinnen die andere Antidiabetika einnahmen oder Nichtdiabetikerinnen ( $24 \%$ vs. $8 \%$ vs. $16 \%, p=0,02$ ) [Jiralerspong $\mathrm{S}$ et al J Clin Oncol.2009; 27:3297-302].
Derzeit untersuchen Pamela J. Goodwin und Kollegen in einer prospektive Phase-III-Studie (MA32) den zusätzlichen Nutzen von Medformin bei der Behandlung von Frauen mit Brustkrebs im Frühstadium.

Peter Leiner

Col NF et al. Metformin and breast cancer risk: a meta-analysis and critical literature review. Breast Cancer Res Treat. 2012;135:639-46.

\section{kurz notiert}

Leitlinie Hyperthermie publiziert

Die Deutsche Gesellschaft für Radioonkologie hat eine neue Leitlinie zur Hyperthermie vorgestellt [Strahlenther Onkol 2012;188 [Suppl 2]: 198-211]. Die regionale Tiefenhyperthermie konzentriert die Wärme $\left(42-43^{\circ} \mathrm{C}\right)$ auf die stark hitzeempfindlichen Krebszellen. Diese setzen Hitzeschockproteine frei, die das Immunsystem alarmieren. Die meist mit Bestrahlung oder Zytostatika kombinierte Therapie wird etwa bei Hochrisiko-Sarkomen, Brustkrebs-Rezidiven, oberflächlichen und Kopf-Hals-Tumoren, Zervix-, Pankreas- sowie Blasenkrebs angewandt. 